\title{
The Implantable Collamer Lens with a central port: review of the literature
}

This article was published in the following Dove Press journal: Clinical Ophthalmology

\author{
Mark Packer \\ Mark Packer MD Consulting, Inc., \\ Boulder, CO, USA
}

Correspondence: Mark Packer

Mark Packer MD Consulting Inc., I 400

Bluebell Ave., Boulder, CO 80302, USA

$\mathrm{Tel}+\mid$ 54| $915029 \mid$

Fax + I 8888409392

Email mark@markpackerconsulting.com

\begin{abstract}
The purpose of this review is to summarize preclinical and clinical data from publications appearing in the peer-reviewed scientific literature relevant to the safety and effectiveness of the EVO Implantable Collamer Lens (ICL) posterior chamber phakic refractive lens with a central port (V4c Visian ICL with KS Aquaport, STAAR Surgical, Inc.). A literature search was conducted using PubMed.gov to identify all articles relating to the EVO ICL. Articles were examined for their relevance, and the references cited in each article were also searched for additional relevant publications. On the basis of a total of 67 preclinical studies and clinical reports, including effectiveness data on 1,905 eyes with average weighted follow-up of 12.5 months and safety data on 4,196 eyes with weighted average follow up of 14.0 months, the EVO ICL is safe and effective for the correction of a broad range of refractive errors. High levels of postoperative uncorrected visual acuity, refractive predictability, and stability demonstrate the effectiveness of the EVO ICL. Safety data suggest reduced rates of anterior subcapsular cataract and pupillary block compared with earlier models. Improved safety and proven effectiveness make EVO an attractive option for surgeons and patients.
\end{abstract}

Keywords: myopia, astigmatism, hyperopia, phakic refractive lens

\section{Introduction}

The EVO Implantable Collamer Lens (ICL Model V4c; STAAR Surgical, Monrovia, CA, USA), which has been commercially available since 2011 , is a single piece posterior chamber phakic refractive intraocular lens designed with a central port to eliminate the need for iridotomy or iridectomy that was required by earlier implantable collamer lens (ICL) models. The central port functions by allowing sufficient aqueous flow from the posterior chamber to the anterior chamber to maintain the normal physiology of the anterior segment of the eye.

\section{Sizing, vault, and safety}

Historically, concerns related to ICL safety have included sizing methodology because of the relationship of excessive or insufficient vault to adverse events such as lens exchange or explantation, pupillary block, endothelial cell loss, pigment dispersion, elevated intraocular pressure (IOP), and cataract. Sizing represents the methodology by which the appropriate overall lens diameter is selected for implantation in order to achieve a safe level of vault, which is the axial distance between the ICL and the crystalline lens. Sizing applies equally to older lens designs without the central port and to the EVO ICL. Authors have suggested alternatives to the most commonly used method of sizing, which is based on the horizontal corneal white-to-white (WTW) distance and the anterior chamber depth (ACD). These alternative methods include the use of ultrasound 
biomicroscopy to measure the sulcus-to-sulcus (STS) distance. However, a meta-analysis of published results has demonstrated that "sulcus-to-sulcus and white-to-white measurementbased sizing methods result neither in clinically meaningful nor statistically significant differences in vault (two-sample two-sided $t$-test using pooled mean and SDs; $t(2,594)=1.33$; $P=0.18) .{ }^{\prime \prime}$ Therefore, the clinical outcomes achieved by the different sizing methodologies appear to be the same.

The degree of variation in vault is independent of sizing methodology and is related to the interaction of the lens implant with the anatomy and physiology of the posterior chamber. As noted, "In summary, neither clinically meaningful nor statistically significant difference in achieved vault differentiates WTW- and STS-based sizing methodologies. No methodology has proven superior to WTW-based sizing in terms of the predictability of vault, and sizing based on WTW and ACD remains the most popular and best-studied technique. While the quest for improved predictability of vault based on different imaging technologies may appear to represent an appealing endeavor, it has resulted neither in a demonstrable reduction in the variability of achieved vault nor in the already low rate of vault-related adverse events that occur following ICL implantation."1

\section{Postapproval study of the visian MICL: implications for safety of the EVO ICL}

Long-term data now available from a Post Approval Study (PAS) of the Implantable Collamer Lens for Myopia (MICL) conducted under the U.S. Food and Drug Administration have provided additional information on safety in regard to the incidence of cataract, elevated IOP, and endothelial cell loss. ${ }^{2}$ The MICL is an earlier version of the ICL, which does not feature a central port and therefore requires the construction of laser iridotomies or a surgical iridectomy to preserve the flow of aqueous from the posterior to the anterior chamber. Nevertheless, the findings of this study regarding vault-related adverse events may be applicable to the EVO ICL, because a meta-analysis has demonstrated that the achieved vault of the EVO ICL and the achieved vault of earlier models without the central port such as the MICL "are not statistically significantly different (two-sample two-sided $t$-test using pooled means and SDs; $t(2,594)=-1.70 ; P=0.09)$. The $95 \%$ confidence interval for the difference (V4c minus others) was found to be -3 to $42 \mu \mathrm{m} . " 1$

\section{Cataract}

In the MICL PAS, the incidence of anterior subcapsular (ASC) cataract was studied in 526 eyes of 294 patients, followed for up to 7.5 years, including 334 eyes available for analysis at 5 or more years. Over that period of time, a total of 31 eyes developed ASC opacities; however, only 5 of these eyes developed visually significant ASC cataracts. Per eye at 5 years, the risk of developing any ASC opacity was $6.1 \%$ and the risk of developing a visually significant ASC cataract was $1.2 \%$. In regard to the incidence of cataract, evidence shows that older age and higher levels of myopia represent risk factors; thus, patient selection can reduce this risk. ${ }^{2}$

The risk of cataract may also be reduced by the new central port design of the EVO ICL. No visually significant cataracts have been reported to date following EVO implantation with up to 5 years of follow-up, and the incidence of nonvisually significant ASC opacities has remained low. ${ }^{1}$ Preclinical studies described below suggest that the central port helps to maintain the health of the crystalline lens by allowing physiologic flow of aqueous humor across the anterior lens capsule.

\section{IOP}

The incidence of elevated IOP requiring treatment, especially in the immediate postoperative period when pupillary block has been reported, is another important safety concern. In the MICL PAS clinical trial, there were 17 cases of pupillary block out of 526 eyes implanted (3.2\%). All cases were treated successfully with neodymium: yttrium aluminum garnet laser iridotomy. ${ }^{2}$ There were also three eyes with elevated IOP due to retained ophthalmic viscosurgical device (viscoelastic, OVD), all of which resolved after irrigation and aspiration. The risk of elevated IOP in the early postoperative period can be mitigated by proper surgical technique because it depends on construction of iridotomies of adequate size and thorough removal of OVD.

The risk of elevated IOP may also be reduced by the central port design: only one case of pupillary block has been reported in the literature we have reviewed, which includes safety data from 4,196 eyes with a weighted average postoperative follow-up of over 1 year.

\section{Endothelial cell loss}

Corneal endothelial health was also addressed in the MICL PAS. Based on clinical data collected through 5-7 years postoperative, the calculated chronic rate of loss of endothelial cell density (ECD) was $\sim 1.8 \%$ per year. Additional data have been provided in the published literature on the MICL. For example, Moya et al have provided a 12-year retrospective study on 144 eyes implanted with the ICL from 1998 to $2001,{ }^{3}$ noting an initial $6.46 \%$ decrease in ECD the first year, 
followed by an annual decrease of $1.20 \%$. These rates should be viewed in the context of the expected age-related loss of ECD, which is $0.6 \%$ per year. ${ }^{4}$ Of note, no cases of corneal decompensation in the absence of trauma have been reported following ICL implantation. ${ }^{5}$ As shown in the literature discussed below, reported rates of endothelial cell loss with EVO are in a range similar to those observed with the MICL.

\section{The EVO ICL}

The EVO ICL Model V4c is a single piece posterior chamber phakic refractive intraocular lens designed with a central convex/concave optical zone diameter of 4.9-5.8 $\mathrm{mm}$ and a $360 \mu \mathrm{m}$ central port. The EVO ICL Model V5 includes a larger optical zone diameter of 5.0-6.1 mm. The central port eliminates the need for iridotomy or iridectomy, which was required by earlier models, because it allows sufficient aqueous flow to maintain the normal physiology of the anterior segment of the eye. The EVO lens is produced from collamer, a proprietary hydroxyethyl methacrylate/porcine-collagen based biocompatible polymer material and an ultraviolet absorbing chromophore.

\section{Literature review}

In order to assess preclinical testing and relevant clinical experience with the EVO ICL when used as described in the Directions for Use, ${ }^{6}$ we performed a literature search with the term "implantable collamer lens" utilizing PubMed.gov. A total of 287 publications were returned and reviewed individually for relevance. Publications were excluded from further review for the following reasons: studies of older ICL models without the central port (128), individual case reports (25), treatment of keratoconus (19), studies of concomitant diagnostic or refractive procedures (13), publications not in English (10), review articles (8), treatment of amblyopia (7), treatment of ametropia following corneal surgery or refractive surgery (5), correspondence (4), treatment of pseudophakic ametropia (4), cataract surgery with ICL explantation (3), and treatment of presbyopia (1). The 60 remaining publications were then selected for examination in detail. References cited in these publications were reviewed for potential relevance and seven publications were additionally reviewed on this basis for a total of 67 peer-reviewed papers examined for this literature review.

\section{Preclinical studies}

Laboratory evaluations of the EVO ICL have focused on aqueous humor fluid dynamics and optical effects of the central port.

\section{Fluid dynamics}

A preclinical study performed by Fujisawa et al demonstrated that introduction of a central $3.0 \mathrm{~mm}$ hole in ICLs implanted in 20 eyes of ten 3-month-old miniature pigs eliminated the development of ASC opacities. ${ }^{7}$ Shiratani et al noted that "unperforated ICLs cause cataracts, but placing a hole in the center of the optic appears to prevent the development of a secondary cataract. In addition, it was found that, even if the hole diameter is only $1.0 \mathrm{~mm}$, cataracts can be prevented. The mechanism of cataract prevention is considered to be related to the aqueous humor circulation."

Kawamorita et al performed a computer simulation of aqueous flow utilizing thermal-hydraulic analysis software. Their results demonstrated increased circulation of aqueous humor to the anterior surface of the crystalline lens when a $0.36 \mathrm{~mm}$ central hole was placed in the ICL, supporting the hypothesis that the central hole "may improve aqueous humor circulation, preventing secondary cataracts" as well as eliminating the requirement for laser iridotomy. ${ }^{9}$ In a second study, these authors determined that the flow around the crystalline lens reached a maximum with a hole size of $\sim 0.4 \mathrm{~mm}$, indicating that "the current model, based on a central hole size of $0.36 \mathrm{~mm}$, was close to ideal." ${ }^{10}$ Recently, these authors also reported that "laser iridotomy is unnecessary from the viewpoint of theoretical aqueous circulation" in the presence of a central hole. ${ }^{11}$ Fernández-Vigo et al reported a full three-dimensional study based on computational fluid dynamics and concluded that "the circulation of aqueous humor is influenced by the type of lens implanted, being more physiological in the central hole model than in the one that requires an iridotomy." 12 These authors more recently provided an interesting theoretical paper describing a model of the circulation of aqueous humor in eyes with and without phakic refractive lenses. ${ }^{13}$

In summary, preclinical studies demonstrate that the $0.36 \mathrm{~mm}$ central port incorporated in the design of EVO provides sufficient aqueous flow to maintain normal fluid dynamics in the eye and improves the circulation of aqueous around the crystalline lens, which may reduce the incidence of cataract formation in comparison to earlier ICL models without the central port.

\section{Optical effects}

Uozato et al investigated the optical performance of the Hole ICL for various ICL powers and effective pupil diameters using a model eye composed of a wet cell filled with balanced salt solution. ${ }^{14}$ Either the conventional ICL or the Hole ICL was placed in the posterior chamber of the model eye. 
The authors concluded that differences in modulation transfer function (MTF) between the Hole ICL and the conventional ICL for various ICL powers and effective pupil diameters were small and clinically negligible. An investigation into the wavefront aberrations of the ICL with and without the central port was conducted by Perez-Vives et al. ${ }^{15}$ No statistically significant differences were found between conventional and Hole ICLs at any refractive power and pupil diameter. The authors concluded that "our study shows good and comparable optical quality of conventional and Hole ICLs for all ICL powers evaluated. ICL decentering affects both ICL models evaluated in the same manner. Although coma aberration increased with ICL decentering, these values were clinically negligible and have no significant effect on the visual performance." Perez-Vives et al used an adaptive optics simulator to study ICL models with and without the central port. ${ }^{16}$ They found no differences $(P>0.05)$ in visual acuity and contrast sensitivity, regardless of decentration.

In an interesting attempt to study the optical effects of a central port design, Ferrer-Blasco et al performed a singleblind cross-over study comparing a standard contact lens to a perforated contact lens designed to mimic the EVO ICL. ${ }^{17}$ The authors concluded that "a contact lens having a central hole provides good visual and optical quality outcomes comparable to those yielded by the same lens without a hole. The results suggest that the impact of a central hole may be clinically negligible."

More recently, Dominguez-Vicent et al investigated the optical properties of the EVO V4c ICL and the EVO V5 ICL, which features an optic diameter up to $6.10 \mathrm{~mm}$ depending on the dioptric power. The authors concluded that "patients with larger pupil diameters could benefit from implantation of the V5 model because this pIOL showed excellent in vitro optical quality with a larger optical diameter than its previous design." 18

Noting that previous investigations had examined only on-axis optical quality, Eppig et al performed an optical simulation to determine whether an ICL model with a central hole would induce more photic phenomena compared to one without a hole in response to off-axis light. ${ }^{19}$ The authors concluded that the central hole may engender stray light and ghost images with off-axis illumination, although on-axis visual quality is unaffected. The authors also noted that "our simulations showed that reflections with negative power ICLs are reduced compared to positive-power ICLs, and that the effect decreases with increasing myopia correction." ${ }^{19}$

In summary, optical studies of the EVO ICL have demonstrated that the presence of the central port does not compromise the optical quality of the image as measured by MTF, wavefront aberrometry, or adaptive optics simulation. One study has shown that reflected stray light from eccentric sources may result in positive dysphotopsia.

\section{Clinical studies: effectiveness}

Measures of clinical effectiveness include postoperative uncorrected distance visual acuity (UDVA), manifest refractive spherical equivalent (MRSE), refractive predictability, and refractive stability. As of October 2018, a total of 27 peer-reviewed papers have provided effectiveness data for the EVO ICL from prospective or retrospective case series, including data from a total of 1,905 eyes with average weighted follow-up of 12.5 months (Table 1).

Considering available data from the literature, including information from 16 publications on 1,023 eyes with weighted average mean preoperative manifest refraction spherical equivalent of $-9.81 \mathrm{D}$ and weighted average follow up of 13.7 months, the reported efficacy index, which is the ratio of uncorrected postoperative visual acuity to preoperative best-corrected acuity, ranges from 0.90 to 1.35 , with a weighted average of 1.04 .

The weighted average uncorrected postoperative visual acuity is $20 / 19(\log M A R-0.02)$, with a range from $20 / 12$ to $20 / 27$ ( $\log$ MAR -0.20 to 0.14 ). The reported percentage of eyes within a half diopter of target averages $90.8 \%$ and ranges from $72 \%$ to $100 \%$, while the percentage of eyes within $1 \mathrm{D}$ of target averages $98.7 \%$ and ranges from $91.8 \%$ to $100 \%$. Selected representative studies are discussed further below.

In a prospective study of 61 eyes of 32 patients implanted with EVO, Yan et al reported, "At 2 years, the spherical equivalent refraction decreased from preoperative $-14.62 \pm 4.29 \mathrm{D}$ to $-0.90 \pm 0.95 \mathrm{D}$, with $79 \%$ of the eyes within $\pm 0.50 \mathrm{D}$ and $98 \%$ within $\pm 1.00 \mathrm{D}$ of the intended correction". ${ }^{20}$

In a multicenter retrospective case series including 351 eyes of 351 subjects, representing the largest single series, eyes were divided into groups based on the preoperative degree of myopia: group 1 included 57 eyes with manifest spherical equivalent less than $-6 \mathrm{D}$, and group 2 included 294 eyes -6 D or more. In this study, Kamiya et al reported, "Uncorrected and corrected visual acuities were $-0.17 \pm 0.14$ and $-0.21 \pm 0.10 \operatorname{logMAR}$ in group 1 , and $-0.16 \pm 0.09$ and $-0.21 \pm 0.08 \log$ MAR in group 2, 1 year postoperatively. In groups 1 and 2, 98\% and $99 \%$ of eyes were within $1.0 \mathrm{D}$ of the targeted correction. Manifest refraction changes of $-0.12 \pm 0.34 \mathrm{D}$ (group 1) and $-0.18 \pm 0.43 \mathrm{D}$ (group 2) occurred from 1 day to 1 year." ${ }^{21}$ The full range of MRSE 
Table I Measures of effectiveness include the efficacy index, uncorrected visual acuity (expressed as logMAR), and the accuracy of refractive correction expressed as percentages within 0.5 and I.0 D of target

\begin{tabular}{|c|c|c|c|c|c|c|c|}
\hline References & $\mathrm{n}$ eyes & N patients & $\begin{array}{l}\text { Follow-up } \\
\text { months }\end{array}$ & $\begin{array}{l}\text { Efficacy } \\
\text { index }\end{array}$ & $\begin{array}{l}\text { Mean logMAR } \\
\text { UDVA }\end{array}$ & $\pm 0.50 \mathrm{D}$ & $\pm \mathbf{I . 0} \mathrm{D}$ \\
\hline Shimizu et al $2012^{48}$ & 20 & 20 & 6 & $1.03 \pm 0.30$ & $-0.20 \pm 0.12$ & $95.0 \%$ & $100.0 \%$ \\
\hline Alfonso et al $2013^{25}$ & 138 & 70 & 6 & 1.00 & $0.009 \pm 0.062$ & $98.5 \%$ & $100.0 \%$ \\
\hline Higueras-Esteban et al $2013^{29}$ & 18 & 10 & 3 & NR & $-0.07 \pm 0.11$ & $100.0 \%$ & $100.0 \%$ \\
\hline Huseynova et al $2014^{23}$ & 44 & 44 & 3 & 1.03 & $0.13 \pm 0.08(0.10-0.30)$ & NR & NR \\
\hline Lisa et al $2015^{24}$ & 147 & 80 & 12 & 1.00 & $0.028 \pm 0.055$ & $93.9 \%$ & $100.0 \%$ \\
\hline Fernández-Vigo et al $2016^{49}$ & 50 & 25 & 3 & NR & $0.05 \pm 0.11$ & NR & NR \\
\hline Liu et al $2016^{43}$ & 82 & 42 & 5 & 1.27 & $-0.03 \pm 0.08$ & NR & NR \\
\hline Shimizu et al $2016^{22}$ & 32 & 32 & 60 & NR & $-0.17 \pm 0.14(0.15-0.30)$ & $88.0 \%$ & $96.0 \%$ \\
\hline Cao et al $2016^{50}$ & 63 & 32 & 6 & $1.11 \pm 0.19$ & $0.118 \pm 0.096$ & NR & NR \\
\hline Cao et al $2016^{51}$ & 78 & 39 & 6 & NR & $0.136 \pm 0.104(0.000-0.301)$ & NR & NR \\
\hline lijima et al $2016^{45}$ & 29 & 29 & 3 & NR & $0.04 \pm 0.21(0.52-0.30)$ & NR & NR \\
\hline \multirow{3}{*}{$\begin{array}{l}\text { Park et al } 2017^{52} \text { (central port } \\
\text { within I, } 2 \text {, or } 3 \text { port diameters } \\
\text { of pupil center) }\end{array}$} & 46 & NR & 3 & NR & $-0.01 \pm 0.06$ & NR & NR \\
\hline & 42 & NR & 3 & NR & $-0.04 \pm 0.07$ & NR & NR \\
\hline & 6 & NR & 3 & NR & $-0.03 \pm 0.08$ & NR & NR \\
\hline Tian et al $2017^{44}$ & 18 & 18 & 1 & NR & -0.02 & NR & NR \\
\hline \multirow{2}{*}{$\begin{array}{l}\text { Kamiya et al } 2017^{21} \\
\text { (myopia }<6, \geq 6 \mathrm{D} \text { ) }\end{array}$} & 57 & 57 & 12 & NR & $-0.17 \pm 0.14(-0.30-0.30)$ & $93.0 \%$ & $98.0 \%$ \\
\hline & 294 & 294 & 12 & NR & $-0.16 \pm 0.09(-0.30-0.15)$ & $94.0 \%$ & $99.0 \%$ \\
\hline Garcia-de La Rosa et al $2018^{53}$ & 76 & 42 & 12 & NR & $0.12 \pm 0.12$ & NR & NR \\
\hline Hyun et al $2017^{54}$ & 24 & 24 & 8 & 1.09 & $0.01 \pm 0.07(-0.10-0.15)$ & NR & NR \\
\hline Fernández-Vigo et al $2017^{31}$ & 54 & 27 & 24 & NR & $0.02 \pm 0.10(0.1-0.2)$ & NR & NR \\
\hline Ganesh et al $2017^{35}$ & 30 & 30 & 12 & 1.12 & $-0.022 \pm 0.021$ & NR & NR \\
\hline Pjano et al $2017^{34}$ & 28 & 16 & 12 & 1.20 & 0.13 & NR & NR \\
\hline Liu et al $2018^{55}$ & 40 & 22 & 3 & NR & $-0.04 \pm 0.05$ & NR & NR \\
\hline Zhao et al $2018^{56}$ & 37 & 19 & 18 & 0.95 & $0.03 \pm 0.05$ & NR & NR \\
\hline Lee et al $2018^{26}$ & 52 & 52 & 6 & $1.35 \pm 0.19$ & NR & $88.0 \%$ & $100.0 \%$ \\
\hline Miao et al $2018^{57}$ & 67 & 38 & 3 & $1.14 \pm 0.23$ & $-0.01 \pm 0.09$ & $72.0 \%$ & $95.0 \%$ \\
\hline Takahashi et al $2018^{58}$ & 42 & 21 & 6 & NR & $-0.03 \pm 0.20$ & $100.0 \%$ & $100.0 \%$ \\
\hline Yan et al $2018^{20}$ & 61 & 32 & 24 & $1.03 \pm 0.23$ & 0.08 & $79.0 \%$ & $98.0 \%$ \\
\hline \multirow[t]{2}{*}{ Kojima et al 2018 (V4c, V5) ${ }^{67}$} & 23 & 23 & 6 & $1.16 \pm 0.22$ & $-0.23 \pm 0.09$ & $94.7 \%$ & $100.0 \%$ \\
\hline & 23 & 23 & 6 & $1.03 \pm 0.23$ & $-0.19 \pm 0.12$ & $84.2 \%$ & $100.0 \%$ \\
\hline $\begin{array}{l}\text { Fernández-Vega-Cueto et al } \\
2018^{36}\end{array}$ & 184 & 92 & 36 & 0.90 & NR & $74.5 \%$ & $91.8 \%$ \\
\hline \multicolumn{2}{|l|}{ Total number of eyes reported } & & \multicolumn{5}{|c|}{ Weighted averages } \\
\hline I,905 & & & 12.5 & 1.04 & -0.02 & $90.8 \%$ & $98.7 \%$ \\
\hline
\end{tabular}

Abbreviations: NR, not reported; UDVA, uncorrected distance visual acuity.

corrected in this study extended from -0.5 to $-18.63 \mathrm{D}$. The authors concluded that " $\ldots$ we believe that the surgical indication for ICL implantation can be expanded to include the correction of low-to-moderate myopia." ${ }^{.21}$

In the study with the longest available follow-up, Shimizu et al reported that "the postoperative UDVAs of the Hole ICL group were 20/20 or better 1, 3, and 6 months and 1,3 , and 5 years postoperatively, in $97 \%, 100 \%, 100 \%$,
$100 \%, 100 \%$, and $85 \%$ of eyes, respectively ..." ${ }^{22}$ Overall, these reports demonstrate excellent postoperative uncorrected visual acuity, accuracy, refractive predictability, and stability up to 5 years postoperatively.

\section{Safety}

Reported safety outcomes include preservation of corrected distance visual acuity (CDVA), changes in IOP, changes 


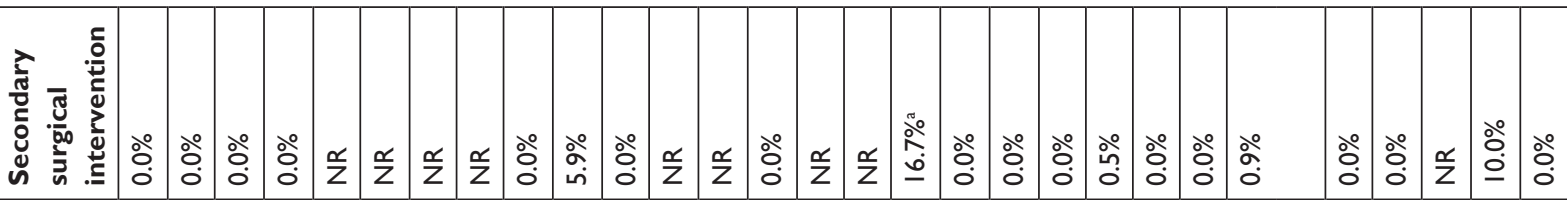

蒫

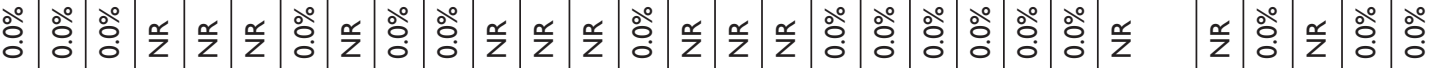

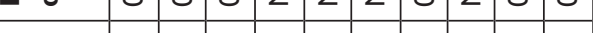

高

O̊ํㅇ 总

(1)

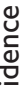

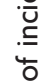

$\ddot{\breve{z}}$

Uే

空

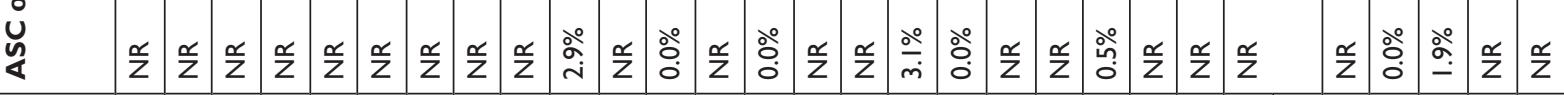
o

$\underline{\overline{\mathrm{d}}}$

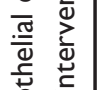

它 $\frac{\overline{0}}{0.0}$

ฮ ज

ํำ

준

这

पे

㟧

बे

ํํㅁ

艺

× $\overline{\mathrm{m}}_{\mathrm{m}} \mathrm{z}$

$\stackrel{.}{5}$

这

究 त्र

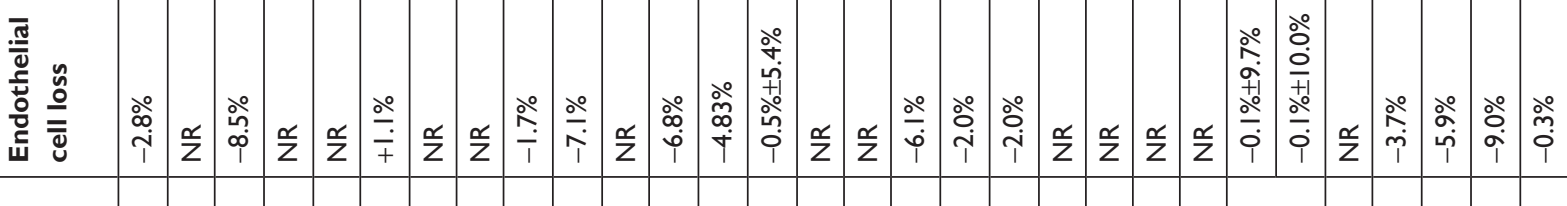

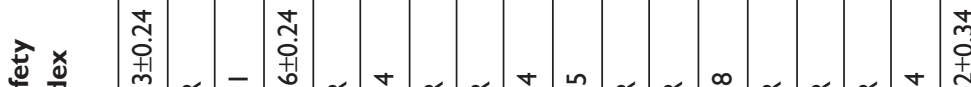

जٓ

인

$\stackrel{5}{\stackrel{2}{0}}$

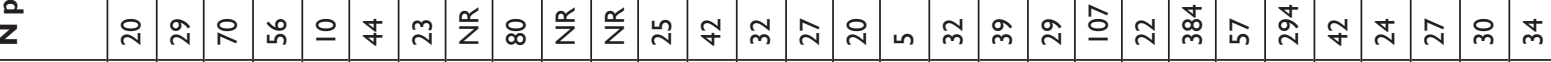

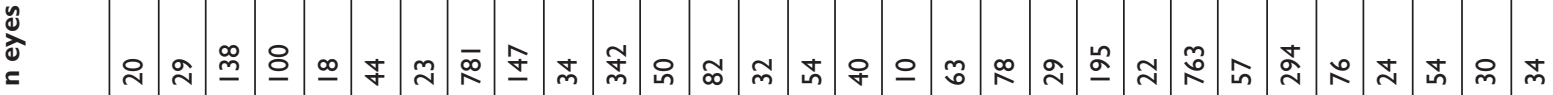

站

ำ

근

六苋

剀

○

y

敢

$\sum \frac{\vec{m}}{>}$

产

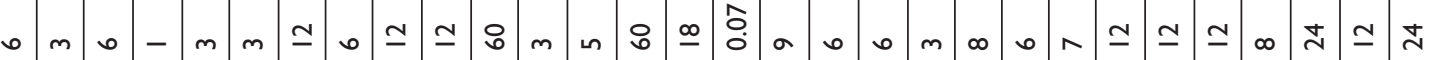

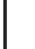

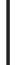

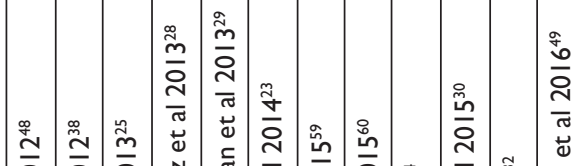

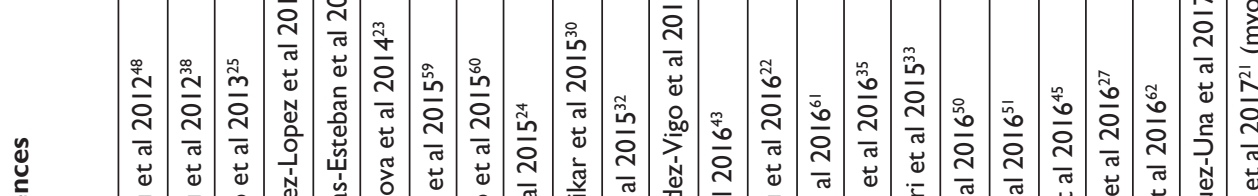

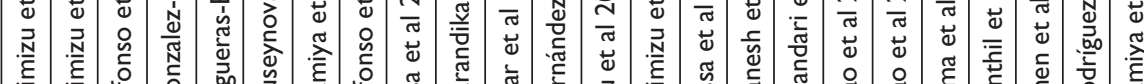

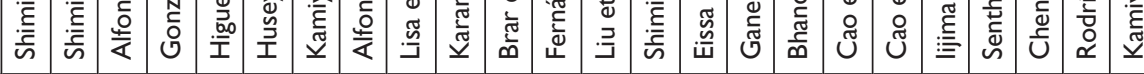




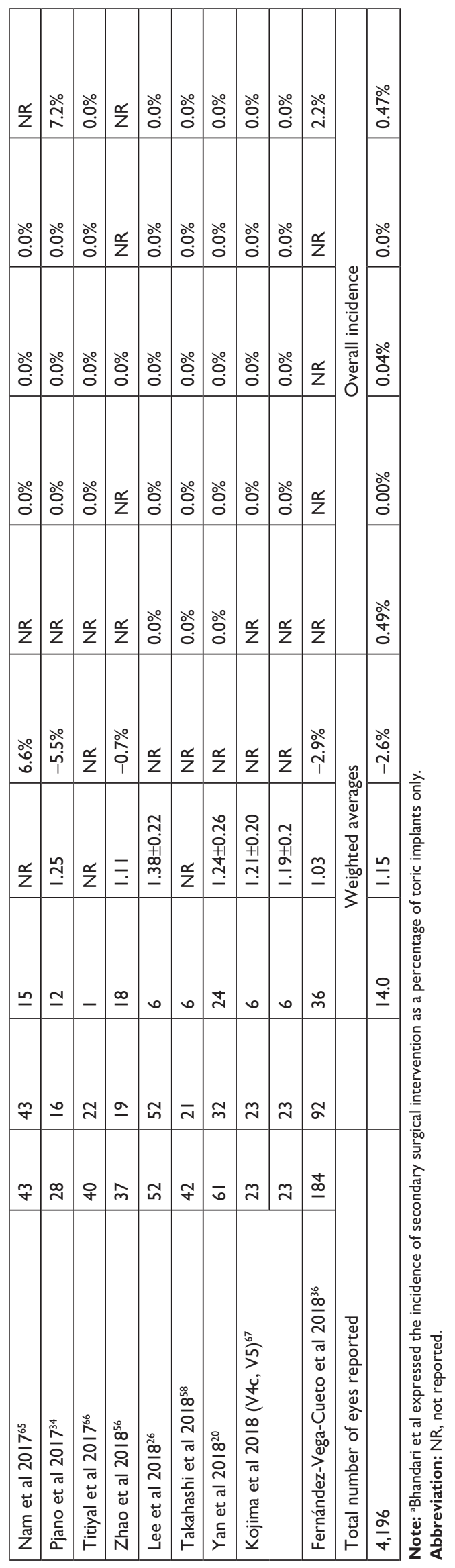

in ECD, ASC opacity or visually significant cataract, and secondary surgical intervention. Table 2 provides a listing of 38 peer-reviewed publications reporting safety data from retrospective or prospective series and includes information on 4,196 eyes with weighted average follow-up of 14.0 months.

\section{CDVA}

The safety index is the ratio of postoperative CDVA to preoperative CDVA. Seventeen publications, including data on 1,100 eyes with a weighted average preoperative manifest refraction spherical equivalent of $-9.60 \mathrm{D}$ and a weighted average follow-up of 13.2 months, demonstrated a weighted average safety index of 1.15, with a range from 1.01 to 1.42 . For example, Huseynova et al noted that "the safety index at 3 months was 1.07 (for group I [V4b]) and 1.14 (for group II [EVO]). ${ }^{{ }^{23} 3}$ Lisa et al, ${ }^{24}$ Alfonso et al, ${ }^{25}$ and Lee et al ${ }^{26}$ reported safety indices of $1.04,1.01$, and $1.38 \pm 0.22$, respectively.

Seventeen studies including a total of 1,410 eyes reported on lines of CDVA lost or gained with a weighted average follow-up of 14.0 months. Only $0.2 \%$ of eyes lost two or more lines of CDVA, while $95.5 \%$ maintained or gained lines of CDVA (Table 3).

\section{IOP}

A single case of pupillary block was reported by Senthil et al, who noted that "the central aquaport of the ICL was blocked with viscoelastic and inflammatory debris.. ${ }^{27}$ One month after surgery CDVA was 20/30 and IOP was $10 \mathrm{mmHg}$ without ocular hypotensive medication. Visual acuity and IOP were maintained at 1 year; however, the pupil remained fixed and dilated. No cases of pigment dispersion glaucoma have been reported.

Additional information on IOP may be gleaned from individual publications. Gonzalez-Lopez et al reported that no eye in their series of 100 eyes had an IOP greater than $30 \mathrm{mmHg}$ at any postoperative measurement. ${ }^{28}$ Higueras-Esteban et al noted "a mild and transient increase in IOP during the first month; however, no chronically elevated IOP levels or pupillary block were observed in either group. ${ }^{{ }^{29}}$ Lisa et al stated that "in the present study, we found no significant rise in IOP $(>20 \mathrm{mmHg})$ in any case, with stable IOP values during the 12-month follow-up .... when we evaluated variations in IOP over time and calculated the change between preoperative and postoperative values, we found that most eyes $(74.1 \%)$ had no IOP variations or showed a reduction of 1-2 mmHg from the preoperative IOP. ${ }^{24}$

\section{ECD}

A total of 21 studies including data on 1,476 eyes with weighted average follow-up of 14.7 months demonstrated 
Table 3 An important measure of safety is the lines of best-corrected visual acuity lost or gained at the study endpoint compared to baseline

\begin{tabular}{|c|c|c|c|c|c|c|c|c|}
\hline References & -3 or more & -2 & -1 & -0.5 & No change & +1 & +2 & +3 or more \\
\hline Shimizu et al $2012^{48}$ & 0 & 0 & 1 & NR & 12 & 5 & 2 & 0 \\
\hline Alfonso et al $2013^{25}$ & 0 & 0 & 0 & NR & 113 & 21 & 4 & 0 \\
\hline Gonzalez-Lopez et al $2013^{28}$ & 0 & 0 & 0 & 3 & \multicolumn{4}{|c|}{97} \\
\hline Lisa et al $2015^{24}$ & 0 & 0 & 0 & NR & 116 & 21 & 5 & 5 \\
\hline Liu et al $2016^{43}$ & 0 & 0 & 0 & NR & 30 & 26 & 18 & 8 \\
\hline Shimizu et al $2016^{22}$ & 0 & 0 & 4 & NR & 9 & 11 & 2 & 0 \\
\hline Bhandari et al $2015^{33}$ & \multicolumn{4}{|c|}{0} & \multicolumn{4}{|c|}{10} \\
\hline Cao et al $2016^{50}$ & \multicolumn{6}{|c|}{0} & & 63 \\
\hline Chen et al $2016^{62}$ & \multicolumn{2}{|l|}{0} & \multicolumn{6}{|c|}{ NR } \\
\hline \multirow[t]{2}{*}{ Kamiya et al $2017^{21}$ (myopia $<6, \geq 6 \mathrm{D}$ ) } & 0 & 0 & 8 & NR & 36 & 13 & 0 & 0 \\
\hline & 0 & 1 & 26 & NR & 142 & 112 & 13 & 0 \\
\hline Garcia-de La Rosa et al $2018^{53}$ & 0 & 1 & 4 & NR & 37 & 19 & 12 & 3 \\
\hline Ganesh et al $2017^{35}$ & \multicolumn{4}{|c|}{0} & \multicolumn{4}{|c|}{30} \\
\hline Pjano et al $2017^{34}$ & 0 & $\mathrm{I}$ & 0 & NR & 11 & 8 & 5 & 3 \\
\hline Lee et al $2018^{26}$ & \multicolumn{4}{|c|}{0} & 7 & \multicolumn{3}{|c|}{45} \\
\hline Takahashi et al $2018^{58}$ & 0 & 0 & 5 & NR & 23 & 12 & 2 & 0 \\
\hline Yan et al $2018^{20}$ & 0 & 0 & 1 & NR & 14 & 33 & 10 & 3 \\
\hline Fernández-Vega-Cueto et al $2018^{36}$ & 0 & 0 & 11 & NR & 145 & 16 & 9 & 3 \\
\hline Total number of eyes reported & \multicolumn{8}{|c|}{ Numbers of eyes that lost or gained lines } \\
\hline 1,410 & 0 & 3 & 57 & 3 & 834 & 343 & 145 & 25 \\
\hline Percentage of eyes that lost or gained lines & $0.0 \%$ & $0.2 \%$ & $4.0 \%$ & $0.2 \%$ & $59.1 \%$ & $24.3 \%$ & $10.3 \%$ & $1.8 \%$ \\
\hline
\end{tabular}

Abbreviation: NR, not reported.

mean endothelial cell loss of $2.6 \%$. It is important to note that this percentage reduction includes both the acute effect of surgery as well as any chronic loss during the follow-up period. A prospective study by Lisa et al of 147 eyes of 80 subjects reported a $1.7 \%$ decline in ECD at 1 year. ${ }^{24}$ In the study with the largest case series, Kamiya et al reported a mean decrease of $0.1 \%$ in 351 eyes of 351 subjects followed for 1 year. ${ }^{21}$ Five-year data from Shimizu et al demonstrate that "the mean percentage of endothelial cell loss 5 years postoperatively was $0.5 \% \pm 5.4 \% . " 22$

\section{ASC opacity and cataract}

Visually significant cataract related to insufficient vault has not been reported in patients implanted with EVO. Overall, 11 publications including data on a total of 617 eyes with a weighted average follow-up of 13 months reported a $0.49 \%$ incidence of asymptomatic ASC opacities. Karandikar et al reported one visually insignificant ASC opacity at 1 year. ${ }^{30}$ Fernández-Vigo et al reported that "we also recorded mild anterior subcapsular cataract in one eye, but CDVA remained stable ( 0.1 logMAR) at 2 years, so the lens was not explanted." ${ }^{11}$ Senthil et al reported one eye that developed localized ASC cataract following pupillary block treated with anterior chamber lavage. ${ }^{27}$
Brar et al have reported on the incidence of cataract over 5 years in 342 eyes implanted with EVO. ${ }^{32}$ The authors noted that "no V4c ICL was explanted due to cataract in this series. This may suggest the benefit of central hole in recent V4c ICL model in providing better nutrition to the natural lens, thus preventing cataract genesis, although $\mathrm{V} 4 \mathrm{c}$ model is launched recently so they have smaller follow-up as compared to the older models." ${ }^{~} 32$

Five years following implantation, Shimizu reported a zero incidence of ASC opacity and cataract. ${ }^{22}$

\section{Secondary surgical intervention}

Data from 28 publications of prospective or retrospective case series demonstrated a $0.47 \%$ incidence of secondary surgical intervention in 2,970 eyes with weighted average follow-up of 16.7 months. Of the 14 total secondary surgical interventions reported, 10 were related to rotation of a toric lens. Karandikar et al described two eyes, ${ }^{30}$ Bhandari et al reported one eye, ${ }^{33}$ Pjano et al reported one eye, ${ }^{34}$ Kamiya et al reported one eye, ${ }^{21}$ Ganesh et al reported three eyes, ${ }^{35}$ and Fernández-Vega-Cueto et al reported two eyes ${ }^{36}$ that required rotation of a toric lens.

In their multicenter study of 351 eyes of 351 subjects followed for 12 months, Kamiya et al noted that "an ICL 
exchange occurred in two eyes $(0.7 \%)$ in group 2 (high myopia $>-6 \mathrm{D})$, due to incorrect initial sizing or power." 21

Pjano et al reported one eye that developed a retinal detachment 3 months after implantation: "The patient had high degenerative myopia and preoperatively underwent prophylactic laser photocoagulation on both eyes. Pars plana vitrectomy was successfully performed, with no further complications on this eye. ${ }^{.34}$ Senthil et al performed anterior chamber lavage to resolve pupillary block related to retained viscoelastic in one eye. ${ }^{27}$

Steinwender et al reported cataract in eleven eyes of eight patients in the immediate postoperative period as a result of an irrigation technique, "... producing an enforced stream through the pIOL hole onto the lens capsule ..." The authors noted that "after changing surgical technique to very gentle irrigation and keeping the cannula near the main incision, more than 90 pIOL implantations were performed in the clinic during a follow-up of 14 months, with no further case of anterior subcapsular cataract occurring." ${ }^{37}$ We have therefore attributed these events to the surgical technique and not to the EVO ICL and have not included these events in the overall rates reported in this review. This report highlights the importance of avoiding operative trauma to the crystalline lens during implantation.

\section{Quality of vision}

Shimizu et al have provided data on higher-order aberrations and contrast sensitivity and found that the central port had no significant impact $(P>0.05) .{ }^{38}$ Kamiya et al reproduced these findings that EVO "implantation appears to be essentially equivalent in the optical quality variables to conventional ICL implantation, suggesting that the presence of the central artificial hole does not significantly affect the optical quality and the intraocular scattering after surgery." 39 Huseynova et al similarly reported no significant differences in wavefront aberrations between EVO and conventional ICL subjects. ${ }^{23}$

\section{Patient-reported outcomes}

Eom et al utilized a modification of a validated questionnaire ${ }^{40}$ and reported that "of 29 total eyes, 18 (62.1\%) experienced glare with the mean duration of $3.0 \pm 3.4$ months (range, 1-12 months), 16 (55.2\%) experienced halos with the mean duration of $3.1 \pm 3.6$ months (range, 1-12 months), $10(34.5 \%)$ experienced starbursts with the mean duration of $1.8 \pm 0.8$ months (range, $1-3$ months) and 15 (51.7\%) experienced ring-shaped dysphotopsia with the mean duration of 2.9 \pm 3.8 months (range, 1-12 months) after hole ICL implantation." ${ }^{41}$ These findings suggest that undesirable visual effects tend to disappear over time. Liu et al offered confirmation in their retrospective study utilizing a different validated questionnaire, ${ }^{42}$ noting that "during the early postoperative follow-up period, halos occurred in 23 patients (54.8\%). With time, halos gradually disappeared at 3 months after operation without any treatments." 43

In their prospective comparative study of lenses with and without the central port, Tian et al reported that "visual fatigue and halo occurred in both groups, visual fatigue occurred in the case of near vision, the incidence of visual fatigue was $25 \%$ in the ICL V4 group and $22.2 \%$ in the ICL V4c group; the incidence of halo was $65 \%$ in the ICL V4 group and $55.6 \%$ in the ICL V4c group. These incidences showed no significant difference between two groups." 44 Overall, patient-reported outcomes reflect the presence of some visual disturbances in the early postoperative period that decrease with time.

Authors have reported high levels of patient satisfaction with EVO implantation. For example, Iijima et al administered the National Eye Institute Refractive Error Quality of Life Instrument-42 to 29 patients at 3 months postoperative and reported a mean satisfaction score of $79.3 .{ }^{45}$ In their 2-year study of 32 patients, Yan et al reported an overall satisfaction score ranging from $0=$ very unsatisfied to $10=$ the most satisfied and noted "All patients were satisfied with their visual performance, and $71 \%$ were more than satisfied (very satisfied). The mean score of overall satisfaction was $9.27 \pm 0.87$ (range, 8-10). And all the patients chose 'yes', when asked 'Would you consider recommending this operation to patients like you?"'20

To date, over 500,000 EVO ICLs with the central port design out of a historic total of 900,000 total ICLs have been distributed globally in countries including Canada, Japan, and those in the European Union, Asia, Latin America, and Africa. Patient satisfaction reported in the manufacturer's registry has remained very high, with $99.4 \%$ of 1,542 patients surveyed stating that they would elect to have the surgery again. ${ }^{46}$

\section{Conclusion}

As McLeod has pointed out, "Phakic intraocular lenses can provide optically superb correction of relatively high degrees of ametropia that lie well beyond the recommended range for keratorefractive procedures, such as laser in situ keratomileusis and photorefractive keratectomy." ${ }^{47}$ High levels of postoperative uncorrected visual acuity, refractive predictability, and stability demonstrate the effectiveness of the EVO ICL. Safety data suggest reduced rates of ASC cataract and pupillary block. Improved safety and effectiveness across a broad range of refractive errors make EVO an attractive option for surgeons and patients. 


\section{Disclosure}

The author is a consultant to STAAR Surgical Company, Advanced Vision Science (Santen Pharmaceutical Co., Ltd.), Alcon Laboratories (Novartis AG), Bausch \& Lomb (Bausch Health Companies Inc.), and Rayner Intraocular Lenses Ltd. The author reports no other conflicts of interest in this work.

\section{References}

1. Packer M. Meta-analysis and review: effectiveness, safety, and central port design of the intraocular collamer lens. Clin Ophthalmol. 2016;10: 1059-1077.

2. Visian ICL (Implantable Collamer Lens) for Myopia: Directions for Use. STAAR Surgical. Available from: http://staar.com/file/ PKG-02012_Rev_2_DFU-for-MICL-Visian-ICL.pdf. Accessed April 3, 2018.

3. Moya T, Javaloy J, Montés-Micó R, Beltrán J, Muñoz G, Montalbán R. Implantable collamer lens for myopia: assessment 12 years after implantation. J Refract Surg. 2015;31(8):548-556.

4. Bourne WM, Nelson LR, Hodge DO. Central corneal endothelial cell changes over a ten-year period. Invest Ophthalmol Vis Sci. 1997;38(3): 779-782.

5. US Department of Health and Human Services; Food and Drug Administration; Center for Devices and Radiological Health; Medical Devices Advisory Committee. Proceedings of the Opthalmic Devices Panel Meeting, March 14, 2014. Available from: https://wayback. archive-it.org/7993/20170114045353/http://www.fda.gov/downloads/ AdvisoryCommittees/CommitteesMeetingMaterials/MedicalDevices/ MedicalDevicesAdvisoryCommittee/OphthalmicDevicesPanel/ UCM392476.pdf. Accessed November 19, 2018.

6. Staar Surgical. SURGICALEVO Visian ICL; Life with EVO; Life with Visian ICL. Available from: https://uk.discovericl.com/life-with-evo. Accessed May 21, 2018.

7. Fujisawa K, Shimizu K, Uga S, et al. Changes in the crystalline lens resulting from insertion of a phakic IOL (ICL) into the porcine eye. Graefes Arch Clin Exp Ophthalmol. 2007;245(1):114-122.

8. Shiratani T, Shimizu K, Fujisawa K, Uga S, Nagano K, Murakami Y. Crystalline lens changes in porcine eyes with implanted phakic IOL (ICL) with a central hole. Graefes Arch Clin Exp Ophthalmol. 2008; 246(5):719-728.

9. Kawamorita T, Uozato H, Shimizu K. Fluid dynamics simulation of aqueous humour in a posterior-chamber phakic intraocular lens with a central perforation. Graefes Arch Clin Exp Ophthalmol. 2012;250(6): 935-939.

10. Kawamorita T, Shimizu K, Shoji N. Effect of hole size on fluid dynamics of a posterior-chamber phakic intraocular lens with a central perforation by using computational fluid dynamics. Graefes Arch Clin Exp Ophthalmol. 2016;254(4):739-744.

11. Kawamorita T, Shimizu K, Shoji N. Theoretical study on the need for laser iridotomy in an implantable collamer lens with a hole using computational fluid dynamics. Eye. 2017;31(5):795-801.

12. Fernández-Vigo JI, Macarro-Merino A, Fernández-Francos J, et al. Computational study of aqueous humor dynamics assessing the vault and the pupil diameter in two posterior-chamber phakic lenses. Invest Ophthalmol Vis Sci. 2016;57(11):4625-4631.

13. Fernández-Vigo JI, Marcos AC, Agujetas R, et al. Computational simulation of aqueous humour dynamics in the presence of a posteriorchamber versus iris-fixed phakic intraocular lens. PLoS One. 2018; 13(8):e0202128.

14. Uozato H, Shimizu K, Kawamorita T, Ohmoto F. Modulation transfer function of intraocular collamer lens with a central artificial hole. Graefes Arch Clin Exp Ophthalmol. 2011;249(7):1081-1085.
15. Pérez-Vives C, Ferrer-Blasco T, Madrid-Costa D, García-Lázaro S, Montés-Micó R. Optical quality comparison of conventional and holevisian implantable collamer lens at different degrees of decentering. Am J Ophthalmol. 2013;156(1):69-76.

16. Pérez-Vives C, Ferrer-Blasco T, Madrid-Costa D, García-Lázaro S, Montés-Micó R. Visual quality comparison of conventional and HoleVisian implantable collamer lens at different degrees of decentering. Br J Ophthalmol. 2014;98(1):59-64.

17. Ferrer-Blasco T, García-Lázaro S, Belda-Salmerón L, AlbarránDiego C, Montés-Micó R. Intra-eye visual function comparison with and without a central hole contact lens-based system: potential applications to ICL design. J Refract Surg. 2013;29(10):702-707.

18. Domínguez-Vicent A, Ferrer-Blasco T, Pérez-Vives C, EsteveTaboada JJ, Montés-Micó R. Optical quality comparison between 2 collagen copolymer posterior chamber phakic intraocular lens designs. J Cataract Refract Surg. 2015;41(6):1268-1278.

19. Eppig T, Spira C, Tsintarakis T, et al. Ghost-image analysis in phakic intraocular lenses with central hole as a potential cause of dysphotopsia. $J$ Cataract Refract Surg. 2015;41(11):2552-2559.

20. Yan Z, Miao H, Zhao F, et al. Two-year outcomes of visian implantable collamer lens with a central hole for correcting high myopia. J Ophthalmol. 2018;2018:1-9.

21. Kamiya K, Shimizu K, Igarashi A. Posterior chamber phakic intraocular lens implantation: comparative, multicentre study in 351 eyes with low-to-moderate or high myopia. Br J Ophthalmol. 2018;102(2):177-181.

22. Shimizu K, Kamiya K, Igarashi A, Kobashi H. Long-term comparison of posterior chamber phakic intraocular lens with and without a central hole (Hole ICL and Conventional ICL) implantation for moderate to high myopia and myopic astigmatism: consort-compliant article. Medicine. 2016;95(14):e3270.

23. Huseynova T, Ozaki S, Ishizuka T, Mita M, Tomita M. Comparative study of 2 types of implantable collamer lenses, 1 with and 1 without a central artificial hole. Am J Ophthalmol. 2014;157(6): $1136-1143$.

24. Lisa C, Naveiras M, Alfonso-Bartolozzi B, Belda-Salmerón L, Montés-Micó R, Alfonso JF. Posterior chamber collagen copolymer phakic intraocular lens with a central hole to correct myopia: one-year follow-up. J Cataract Refract Surg. 2015;41(6):1153-1159.

25. Alfonso JF, Lisa C, Fernández-Vega Cueto L, Belda-Salmerón L, Madrid-Costa D, Montés-Micó R. Clinical outcomes after implantation of a posterior chamber collagen copolymer phakic intraocular lens with a central hole for myopic correction. J Cataract Refract Surg. 2013;39(6): 915-921.

26. Lee H, Kang DSY, Choi JY, et al. Rotational stability and visual outcomes of V4c toric phakic intraocular lenses. J Refract Surg. 2018; 34(7):489-496.

27. Senthil S, Choudhari NS, Vaddavalli PK, Murthy S, Reddy JC, Garudadri CS. Etiology and management of raised intraocular pressure following posterior chamber phakic intraocular lens implantation in myopic eyes. PLoS One. 2016;11(11):e0165469.

28. Gonzalez-Lopez F, Bilbao-Calabuig R, Mompean B, et al. Intraocular pressure during the early postoperative period after 100 consecutive implantations of posterior chamber phakic intraocular lenses with a central hole. J Cataract Refract Surg. 2013;39(12):1859-1863.

29. Higueras-Esteban A, Ortiz-Gomariz A, Gutiérrez-Ortega R, et al. Intraocular pressure after implantation of the Visian Implantable Collamer Lens with CentraFLOW without iridotomy. Am J Ophthalmol. 2013;156(4):800-805.

30. Karandikar S, Bhandari V, Reddy J. Outcomes of implantable collamer lens V4 and V4c for correction of high myopia - a case series. Nepal J Ophthalmol. 2015;7(14):164-172.

31. Fernández-Vigo JI, Macarro-Merino A, Fernández-Vigo C, et al. Impacts of implantable collamer lens $\mathrm{V} 4 \mathrm{c}$ placement on angle measurements made by optical coherence tomography: two-year follow-up. Am J Ophthalmol. 2017;181:37-45. 
32. Brar S, Ganeresh S, Pandey R. Incidence \& factors responsible for Implantable Collamer Lens (ICL) explantation \& outcomes of further management - 5 year retrospective study. EC Ophthalmology. 2015; 3(1):231-239.

33. Bhandari V, Karandikar S, Reddy JK, Relekar K. Implantable collamer lens V4b and V4c for correction of high myopia. J Curr Ophthalmol. 2015;27(3-4):76-81.

34. Pjano MA, Pidro A, Biscevic A, Grisevic S, Pandzic B, Cerovic V. Refractive outcomes of posterior chamber phakic intraocular lens implantation for correction of myopia and myopic astigmatism. Med Arch. 2017;71(2):93-96.

35. Ganesh S, Brar S, Pawar A. Matched population comparison of visual outcomes and patient satisfaction between 3 modalities for the correction of low to moderate myopic astigmatism. Clin Ophthalmol. 2017;11 1253-1263.

36. Fernández-Vega-Cueto L, Lisa C, Esteve-Taboada JJ, Montés-Micó R, Alfonso JF. Implantable collamer lens with central hole: 3-year follow-up. Clin Ophthalmol. 2018;12:2015-2029.

37. Steinwender G, Varna-Tigka K, Shajari M, Kohnen T. Anterior subcapsular cataract caused by forceful irrigation during implantation of a posterior chamber phakic intraocular lens with a central hole. J Cataract Refract Surg. 2017;43(7):969-974.

38. Shimizu K, Kamiya K, Igarashi A, Shiratani T. Intraindividual comparison of visual performance after posterior chamber phakic intraocular lens with and without a central hole implantation for moderate to high myopia. Am J Ophthalmol. 2012;154(3):486-494.

39. Kamiya K, Shimizu K, Saito A, Igarashi A, Kobashi H. Comparison of optical quality and intraocular scattering after posterior chamber phakic intraocular lens with and without a central hole (Hole ICL and Conventional ICL) implantation using the double-pass instrument. PLoS One. 2013;8(6):e66846.

40. Mcalinden C, Pesudovs K, Moore JE. The development of an instrument to measure quality of vision: the Quality of Vision (QoV) questionnaire. Invest Ophthalmol Vis Sci. 2010;51(11):5537-5545.

41. Eom Y, Kim DW, Ryu D, et al. Ring-shaped dysphotopsia associated with posterior chamber phakic implantable collamer lenses with a central hole. Acta Ophthalmol. 2017;95(3):e170-e178.

42. Mcghee CN, Craig JP, Sachdev N, Weed KH, Brown AD. Functional, psychological, and satisfaction outcomes of laser in situ keratomileusis for high myopia. J Cataract Refract Surg. 2000;26(4):497-509.

43. Liu T, Linghu S, Pan L, Shi R. Effects of V4c-ICL implantation on myopic patients' vision-related daily activities. J Ophthalmol. 2016; 2016:5717932

44. Tian Y, Jiang HB, Jiang J, Wen D, Xia XB, Song WT. Comparison of implantable collamer lens visian ICL V4 and ICL V4c for high myopia: a cohort study. Medicine. 2017;96(25):e7294.

45. Iijima A, Shimizu K, Yamagishi M, Kobashi H, Igarashi A, Kamiya K. Assessment of subjective intraocular forward scattering and quality of vision after posterior chamber phakic intraocular lens with a central hole (Hole ICL) implantation. Acta Ophthalmol. 2016;94(8):e716-e720.

46. Staar Surgical. Patient Survey; Visian ICL ${ }^{\mathrm{TM}}$ (Implantable Collamer Lens) For Nearsightedness; Facts You Need To Know About STAAR Surgical's Visian ICL; Surgery; Patient Information Booklet. Available from: https://www.staar.com/our-resources. Accessed November 19 2018.

47. Mcleod SD. Long-term clinical outcomes and cataract formation rates after posterior phakic lens implantation for myopia. JAMA Ophthalmol. 2016;134(5):494-495.

48. Shimizu K, Kamiya K, Igarashi A, Shiratani T. Early clinical outcomes of implantation of posterior chamber phakic intraocular lens with a central hole (Hole ICL) for moderate to high myopia. Br J Ophthalmol. 2012;96(3):409-412.

49. Fernández-Vigo JI, Macarro-Merino A, Fernández-Vigo C, et al. Effects of implantable collamer lens V4c placement on iridocorneal angle measurements by Fourier-domain optical coherence tomography. Am J Ophthalmol. 2016;162:43-52.
50. Cao X, Wu W, Wang Y, Xie C, Tong J, Shen Y. Posterior chamber collagen copolymer phakic intraocular lens with a central hole for moderateto-high myopia: first experience in China. Medicine. 2016;95(36): e4641.

51. Cao X, Wu W, Wang Y, Xie C, Shen Y. Comparison over time of vault in Chinese eyes receiving implantable contact lenses with or without a central hole. Am J Ophthalmol. 2016;172:111-117.

52. Park MJ, Jeon HM, Lee KH, Han SY. Comparison of postoperative optical quality according to the degree of decentering of V4c implantable collamer lens. Int J Ophthalmol. 2017;10(4):619-623.

53. Garcia-de La Rosa G, Olivo-Payne A, Serna-Ojeda JC, et al. Anterior segment optical coherence tomography angle and vault analysis after toric and non-toric implantable collamer lens V4c implantation in patients with high myopia. Br J Ophthalmol. 2018;102(4):544-548.

54. Hyun J, Lim DH, Eo DR, Hwang S, Chung ES, Chung TY, Dr E. A comparison of visual outcome and rotational stability of two types of toric implantable collamer lenses (TICL): V4 versus V4c. PLoS One. 2017;12(8):e0183335.

55. Liu HT, Zhou Z, Luo WQ, et al. Comparison of optical quality after implantable collamer lens implantation and wavefront-guided laser in situ keratomileusis. Int J Ophthalmol. 2018;11(4):656-661.

56. Zhao J, Luo D, Sun Y, et al. Implanting a posterior chamber phakic intraocular lens in highly myopic eyes with peripheral primary iris and ciliary body cysts. Eur J Ophthalmol. 2018;1:112067211876644.

57. Miao H, Chen X, Tian M, Chen Y, Wang X, Zhou X. Refractive outcomes and optical quality after implantation of posterior chamber phakic implantable collamer lens with a central hole (ICL V4c). BMC Ophthalmol. 2018;18(1):141.

58. Takahashi M, Kamiya K, Shoji N, Kato S, Igarashi A, Shimizu K. Intentional undercorrection by implantation of posterior chamber phakic intraocular lens with a central hole (Hole ICL) for early presbyopia. Biomed Res Int. 2018;2018:1-5.

59. Kamiya K, Shimizu K, Ando W, Igarashi A, Iijima K, Koh A. Comparison of vault after implantation of posterior chamber phakic intraocular lens with and without a central hole. J Cataract Refract Surg. 2015;41(1):67-72.

60. Alfonso JF, Lisa C, Fernández-Vega L, Almanzar D, Pérez-Vives C, Montés-Micó R. Prevalence of cataract after collagen copolymer phakic intraocular lens implantation for myopia, hyperopia, and astigmatism. J Cataract Refract Surg. 2015;41(4):800-805.

61. Eissa SA, Sadek SH, El-Deeb MW. Anterior chamber angle evaluation following phakic posterior chamber collamer lens with centraFLOW and its correlation with ICL vault and intraocular pressure. J Ophthalmol. 2016;2016:1383289

62. Chen H, Niu G, Fan Y, Ma J. Comparison of intraocular pressure andvault after implantation of implantable collamer lens with and without a central hole. BMC Ophthalmol. 2016;16(1):203.

63. Rodríguez-Una I, Rodríguez-Calvo PP, Fernández-Vega Cueto L, Lisa C, Fernández-Vega Cueto A, Alfonso JF. Intraocular pressure after implantation of a phakic collamer intraocular lens with a central hole. J Refract Surg. 2017;33(4):244-249.

64. Goukon H, Kamiya K, Shimizu K, Igarashi A. Comparison of corneal endothelial cell density and morphology after posterior chamber phakic intraocular lens implantation with and without a central hole. $B r J$ Ophthalmol. 2017;101(11):1461-1465.

65. Nam SW, Lim DH, Hyun J, Chung ES, Chung TY. Buffering zone of implantable collamer lens sizing in V4c. BMC Ophthalmol. 2017; 17(1):260.

66. Titiyal JS, Kaur M, Sahu S, Sharma N, Sinha R. Real-time assessment of intraoperative vaulting in implantable collamer lens and correlation with postoperative vaulting. Eur J Ophthalmol. 2017;27(1): $21-25$.

67. Kojima T, Kitazawa Y, Nakamura T, et al. Prospective randomized multicenter comparison of the clinical outcomes of V4c and V5 implantable collamer lenses: a contralateral eye study. J Ophthalmol. 2018;2018:7623829 


\section{Publish your work in this journal}

Clinical Ophthalmology is an international, peer-reviewed journal covering all subspecialties within ophthalmology. Key topics include: Optometry; Visual science; Pharmacology and drug therapy in eye diseases; Basic Sciences; Primary and Secondary eye care; Patient Safety and Quality of Care Improvements. This journal is indexed on

Submit your manuscript here: http://www.dovepress.com/clinical-ophthalmology-journal
PubMed Central and CAS, and is the official journal of The Society of Clinical Ophthalmology (SCO). The manuscript management system is completely online and includes a very quick and fair peer-review system, which is all easy to use. Visit http://www.dovepress.com/ testimonials.php to read real quotes from published authors. 